Musées, Patrimoine et Culture scientifiques et techniques

$110 \mid 2007$

mars - avril 2007

\title{
Le jonglage objet-cartel
}

\section{Anne-Sophie Grassin}

URL : http://journals.openedition.org/ocim/776

DOI : $10.4000 /$ ocim. 776

ISSN : 2108-646X

\section{Éditeur}

OCIM

Édition imprimée

Date de publication : 1 mars 2007

Pagination : 4-12

ISSN : 0994-1908

Référence électronique

Anne-Sophie Grassin, «Le jonglage objet-cartel », La Lettre de l'OCIM [En ligne], 110 | 2007, mis en ligne le 15 mars 2011, consulté le 01 mai 2019. URL : http://journals.openedition.org/ocim/776 ; DOI :

10.4000/ocim. 776

Tous droits réservés 


\section{Le jonglage objet-cartel}

\section{Anne-Sophie Grassin *}

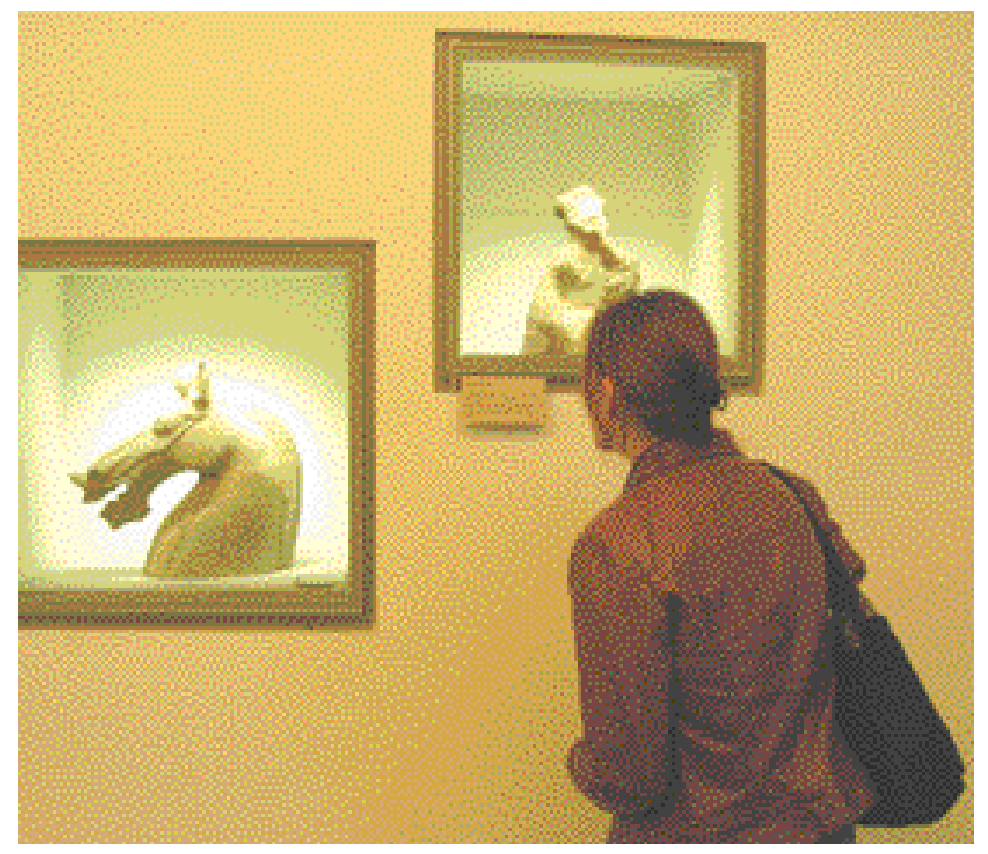

Visiteuse jonglant entre un objet et son cartel (c) Musée national des Arts asiatiques Guimet

* Anne-Sophie Grassin est diplômée en Recherche Appliquée en Muséologie et travaille au service des collections du musée des Civilisations de l'Europe et de la Méditerranée anne-sophie.grassin@culture.gouv.fr
L'étude du comportement des visiteurs face aux cartels des expositions permet à l'auteur d'analyser la structuration de l'information contenue dans les textes de ces cartels, de définir un modèle qui optimise l'approche de l'objet et de montrer les stratégies de visite et les processus interprétatifs qui découlent de ce comportement.

Le jonglage objet-cartel est le mouvement de va-etvient qu'un visiteur exécute plusieurs fois entre l'observation d'un objet et la lecture de son cartel. En salle d'exposition, cette attitude est récurrente. Le jonglage est une démarche d'appropriation que les concepteurs d'expositions pourraient prendre en compte pour maintenir le visiteur en lien avec le discours de l'exposition.

\section{Qu'entend-on par " jonglage "?}

Vous, visiteurs de musées, spécialistes ou néophytes, avez tous au moins une fois été interpellé par un objet exposé, un objet singulier mis à l'honneur et présenté là, juste devant vous. Était-ce un aspect de l'objet, un mot, une phrase inscrite sur l'étiquette à proximité de cet objet ? Était-ce une ambiance, une atmosphère particulière qui enveloppe le discours de l'exposition ? Était-ce un sentiment personnel, un souvenir ?... Peu importe, vous avez eu envie d'en savoir plus sur cet ensemble qui pique votre curiosité. Vous avez éprouvé le besoin de passer un peu de temps à comprendre cet objet, à aller et venir 
entre l'information contenue dans le cartel et l'objet même. Si vous avez déjà vécu cette scène ou si vous avez été témoin de cette relation entre un visiteur et un objet, alors vous connaissez déjà en partie le comportement du « jonglage».

Le jonglage a été identifié au cours d'une investigation de plus grande envergure sur l'influence du média exposition sur le fonctionnement psychologique du visiteur adulte, mise en place par le groupe de recherche sur les musées et l'éducation des adultes de l'université de Montréal. L'évènement qui a permis l'analyse du jonglage est Xían, capitale éternelle, une exposition d'archéologie chinoise présentée au musée de la Civilisation à Québec en 2002.

\section{Le jonglage objet-cartel et les publications muséologiques}

Le jonglage tel que nous l'étudions est abordé dans les textes évoquant l'étroite et complexe relation qui peut se tisser entre un visiteur et un objet dans l'espace de l'exposition. Toutefois, le phénomène de jonglage n'est jamais nommé.

À la lumière des publications muséologiques, le jonglage objet-cartel apparaît comme un processus mental qui intervient au cours de la visite muséale, lors de la confrontation du visiteur à l'objet. En s'inspirant du texte de Jacobi et Poli (1991), on peut penser qu'il s'agit d'un travail particulier de va-et-vient de l'objet aux signes linguistiques et vice-versa. Ce travail est d'abord celui de l'œil, qui commence par percevoir l'expôt et son étiquette comme une entité globale, et c'est par un renvoi de l'un à l'autre que le visiteur instruirait un travail intellectuel (Desjardins et Jacobi 1992) ; le jonglage est donc une opération mentale, une stratégie cognitive. Quel est vraiment ce renvoi ? Filiatrault (1998) a suggéré que les habiletés intellectuelles mises en œuvre dans l'appropriation des objets étaient souvent liées à un mouvement de va-et-vient inscrit dans des séquences complexes ; le va-et-vient objet-cartel pourrait alors s'inscrire dans un processus d'apprentissage.

Le jonglage objet-cartel peut être considéré comme un moyen ou une technique qui permet au visiteur de développer une analyse détaillée des objets muséaux. En effet à travers ce qu'il nomme « a viewing process », Lowe (2000) explique que l'information écrite dans l'exposition doit conduire le regard du visiteur et être combinée à un processus de construction de sens. D'après Poli (1992) le renvoi du texte à l'objet ou de l'objet au texte traduit la relation que le visiteur entretient avec le concepteur. Et le travail de O'Neill (1998) porte à croire que plus le lien entre l'objet et le cartel est explicité, démontré, plus l'interprétation personnelle du visiteur est en liaison avec celle que propose l'institution. Pour ce faire, Serrell (1996) explique que les mots de l'étiquette et l'expérience de l'observateur doivent fonctionner ensemble. Ce principe d'interaction entre le verbe, l'image et le visiteur est de créer une expérience complète que ni l'écrit ni l'objet ne pourrait faire seuls. Enfin, les analyses de O'Neill et Dufresne-Tassé (2000) corroborent les idées précédemment évoquées : il semble que ce va-et-vient soit une manière d'associer plusieurs éléments entre eux pour tirer du sens de ce qui est présent devant soi. C'est une dynamique de rapprochement entre un écrit et un objet, une opération mentale réalisée par le visiteur par association des éléments observés pour construire du sens. Cette utilisation simultanée de l'objet et du texte de l'étiquette serait donc un moyen d'impliquer le visiteur en lui permettant d'accroître son pouvoir d'interprétation des objets, étape nécessaire à l'appropriation du discours de l'exposition.

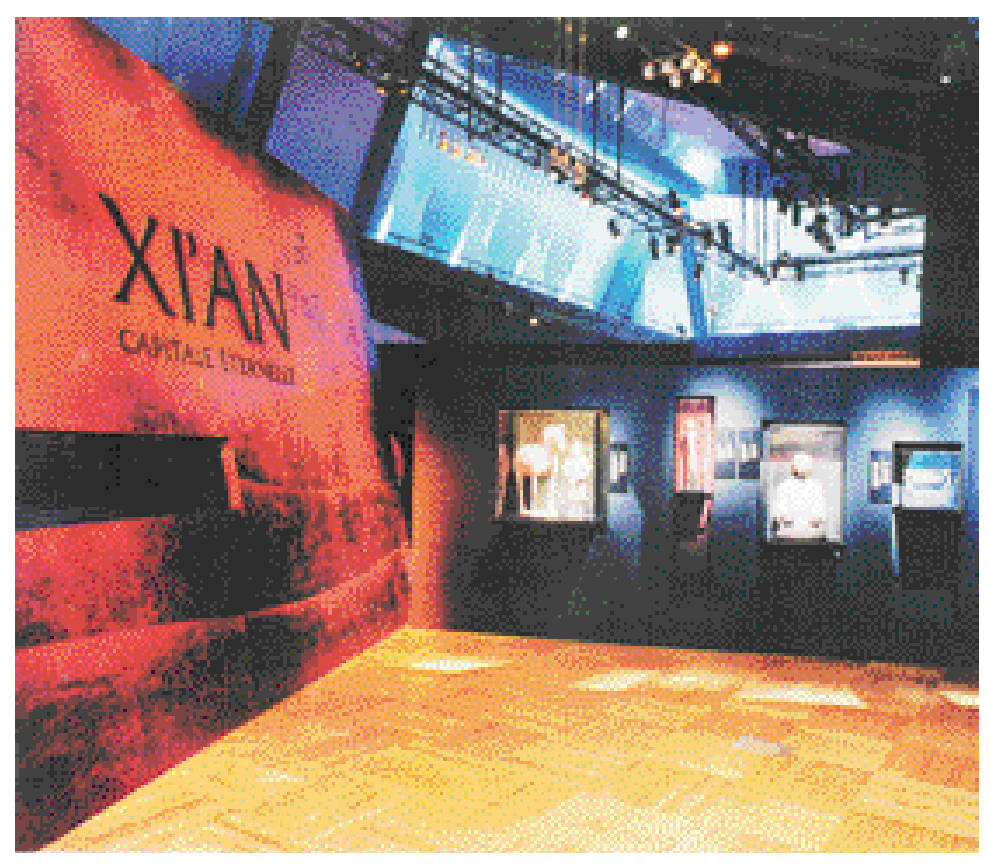

Espace introductif de $X_{i}$ 'an, capitale éternelle (c) Musée de la Civilisation, Québec/Jacques Lessard

Pour conclure sur ce point, il est intéressant de noter que ce que nous appelons le jonglage objet-cartel trouve un réel écho chez Poli (2002), qui évoque des "phénomènes d'intercompréhension sémantique » au musée. Ces phénomènes s'opéreraient chez le visiteur entre le registre langagier et les autres registres 
expographiques. Le va-et-vient entre un objet et son étiquette serait en effet une interaction entre la médiation verbale, véhiculée par le texte du cartel, et la médiation non verbale émanant du registre de l'objet. De ce qui précède, il ressort que :

- la notion même de jonglage est absente des textes muséologiques ; toutefois, cette notion y trouve de nombreux appuis ;

- cette absence indique que les phénomènes d'intercompréhension sémantique ont été peu problématisés au musée.

\section{Les causes du jonglage : un questionnement phare}

Il est intéressant de noter que les deux études consacrées à l'interaction entre un objet et un cartel ont été réalisées par des linguistes questionnant le champ muséal (Bosredon, 1997 ; Kawashima et Poli, 2000). En effet, ces études ne cherchent pas à expliquer le processus impliqué dans le va-et-vient objetcartel. Elles n'envisagent pas le phénomène dans son ensemble. Elles s'attachent plutôt à deux de ses effets : l'identification et la réutilisation des titres des cartels lorsque les visiteurs observent les objets. Aucune étude n'existe donc sur ce qui pourrait déclencher le besoin d'aller et venir plusieurs fois entre un objet et une étiquette. Souhaitant pallier cette lacune, nous avons travaillé sur l'influence potentielle des éléments en présence. Quatre éléments nous paraissent susceptibles d'être les déclencheurs du jonglage objet-cartel : la nature des objets, la situation muséale, le visiteur, le cartel.

Dans une certaine mesure, le jonglage objet-cartel est une modalité de lecture. D’après Jacobi et Poli (1991) puis O’Neill et Dufresne-Tassé (2000), la forme du cartel pourrait avoir une incidence sur la manière d'approcher l'objet, et pourrait faciliter le va-et-vient avec l'objet.

\section{Méthode}

Le jonglage faisant partie de l'expérience immédiate du visiteur en situation d'observation, nous avons recueilli le discours de ces individus au cours de leur visite, au moyen d'une consigne inspirée de l'approche appelée «Thinking Aloud» (penser tout haut). Cette méthode empruntée aux sciences cognitives (Ericcson et Simon, 1993), a été adaptée à la situation muséale et validée pour cette situation par Dufresne-Tassé (Dufresne-Tassé, Sauvé, Weltz-Fairchild, Banna, Lepage et Dassa, 1998). Cette façon d'obtenir de l'information consiste à demander à chaque visiteur participant à l'étude d'exprimer à voix haute ce qu'il pense et ressent au fur et à mesure de sa visite. Un chercheur l'accompagne et observe discrètement son comportement, enregistre son discours, et note son parcours. Les données enregistrées sont retranscrites afin d'être analysées dans leur forme écrite.

Un échantillon de 86 visiteurs a donc été constitué en fonction des habitudes de fréquentation muséale. Il comprend un nombre semblable d'étudiants, de personnes au travail, et de retraités des deux sexes, possédant trois niveaux de formation. Afin de comprendre la complexité de ce phénomène de jonglage objet-cartel, une grille d'analyse a été élaborée. Elle met en relief les objets et les cartels sur lesquels les visiteurs s'arrêtent pour jongler et facilite le repérage des situations précises de jonglage.

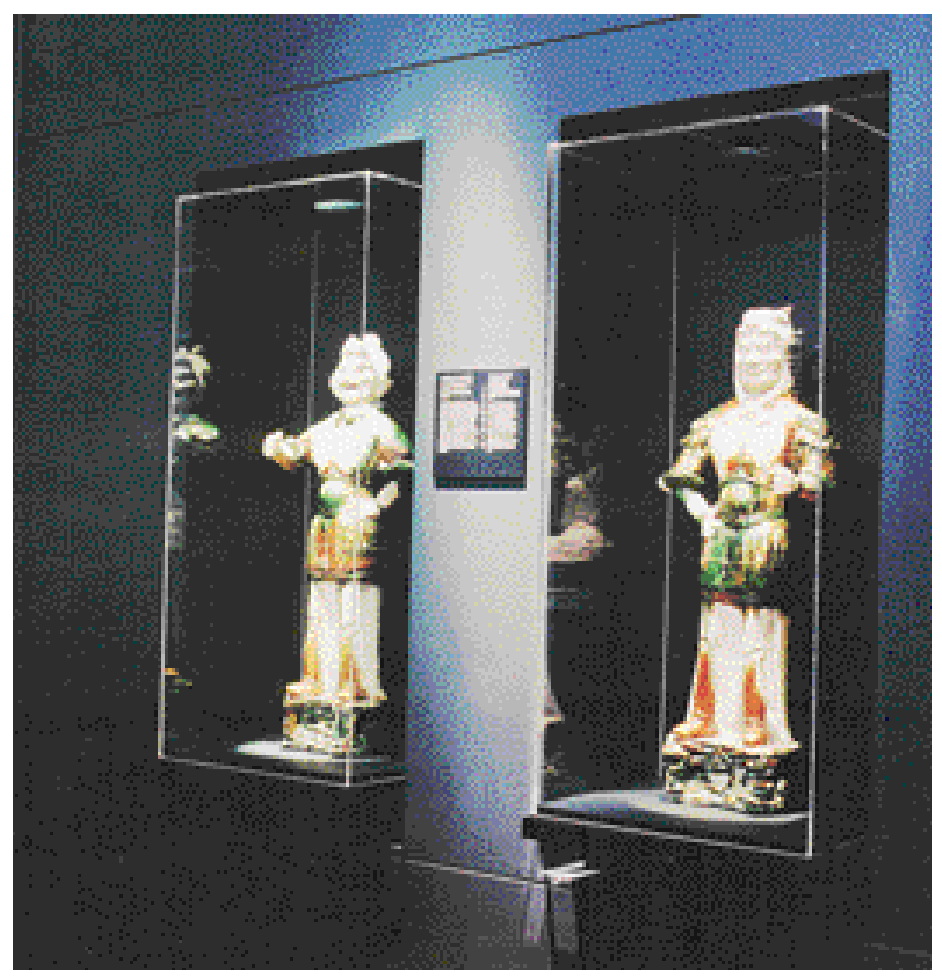

$X i^{\prime} a n$, capitale éternelle. Une étiquette prédicative entre deux gardiens de tombeau, sculptures en terre cuite et glaçure tricolore, de la dynastie des Tang, objets sur lesquels les visiteurs ont beaucoup jonglé. ○ Musée de la Civilisation, Québec/Jacques Lessard

\section{Des résultats surprenants}

Les résultats obtenus dans l'exposition Xi'an, capitale éternelle montrent que les visiteurs ont fortement eu tendance à jongler. En effet, 68 visiteurs sur 86 approchent l'objet par le jonglage avec un cartel, ce qui représente $79 \%$ de la population étudiée. Ainsi, le fait 
d'associer l'observation de l'objet à l'étiquette qui l'accompagne semble tenir une place essentielle dans le déroulement de la visite, et parait déterminant dans l'approche des objets par l'ensemble des visiteurs. Cette tendance confirme les impressions notées en suivant pas à pas chacun des sujets dans l'exposition.

Pour identifier les causes de ce comportement, nous avons analysé l'incidence potentielle des quatre éléments en jeu dans ce comportement.

Nous avons tout d'abord considéré les matériaux constitutifs des objets exposés, leurs tailles et leurs fonctions afin d'évaluer l'incidence de la nature des objets dans le déclenchement du jonglage objet-cartel. Le matériau constitutif est peu déterminant dans le jonglage, même si l'or et le grès sont des matériaux sur lesquels les va-et-vient sont nombreux. Les tests statistiques indiquent en effet que les écarts entre les moyennes de jonglages ne sont pas significatifs. De même, la taille des objets a une faible incidence sur le jonglage. La fonction première des objets influerait également peu sur le jonglage, même si certaines d'entre elles inciteraient davantage les visiteurs à utiliser concurremment les objets et leurs étiquettes. Certains objets peuvent en effet provoquer un va-et-vient objet-étiquette par la curiosité que suscite leur apparence. La rencontre objet-visiteur peut prendre la forme d'une contemplation et d'un désir de mieux connaître l'objet. La démarche de lecture du cartel répond alors à cette demande et permet en outre d'enrichir la contemplation esthétique d'une explication scientifique.

Un autre facteur potentiellement influent dans le jonglage est la " situation muséale ». L'examen de la « situation muséale », l'ordonnancement général et physique du discours, a montré deux choses :

- le jonglage n'est pas induit par le nombre d'objets exposés par partie, ni par la présence ou non de regroupements d'objets. Les tests statistiques indiquent que les écarts entre les moyennes de jonglages ne sont pas significatifs ;

- il n'y a pas non plus d'incidence de l'exposition ou non de l'objet sous vitrine, car là encore, les écarts entre les moyennes de jonglages ne sont pas significatifs.

Par contre, il semblerait que le jonglage soit le fait d'une population plutôt instruite, habituée des musées et sortie de l'activité professionnelle.

Enfin, les données obtenues dans la partie consacrée à l'analyse des cartels sont les plus riches : la rédaction du texte des cartels a une incidence sur le jonglage. Les données obtenues montrent que ce qui fait jongler tient plus à la manière dont on parle de l'objet qu'à l'objet lui-même.

\section{" Il y a une description sur l'étiquette et je recherche cette description dans l'objet que je regarde "(1)}

Pour vérifier l'hypothèse selon laquelle la structure interne du texte des étiquettes peut favoriser le vaet-vient du regard entre l'objet et l'étiquette, il est nécessaire d'analyser la composition des textes des cartels de Xi'an, capitale éternelle. 93 cartels sur 97 possèdent un texte sous le titre ; on dit qu'ils sont allongés ou prédicatifs. 4 seulement ne possèdent pas de texte, leurs caractéristiques sont simplement listées sous forme de points non rédigés ; ce sont des cartels « identificatoires » ou autonymes. L'examen de la structuration interne de l'information contenue dans les textes de ces cartels nous a permis de distinguer deux groupes

- les cartels du groupe A sont les cartels sur lesquels les visiteurs jonglent le plus,

- les cartels du groupe B sont les cartels sur lesquels les visiteurs jonglent le moins.

Les 4 cartels autonymes se trouvent dans les cartels du groupe B. La présence d'un texte sous le titre de l'œuvre jouerait-elle un rôle de déclenchement du jonglage ? Pour le savoir nous avons découpé l'information contenue dans ces textes en "énoncés ", c'est-à-dire en unités de textes ou portions de phrases. Nous en avons défini douze :

- la dénomination confère une existence à l'objet en lui donnant un nom, un titre, une identification ;

- la dénomination « qualifiée " précise l'identité de l'objet par la présence d'un adjectif qualificatif ;

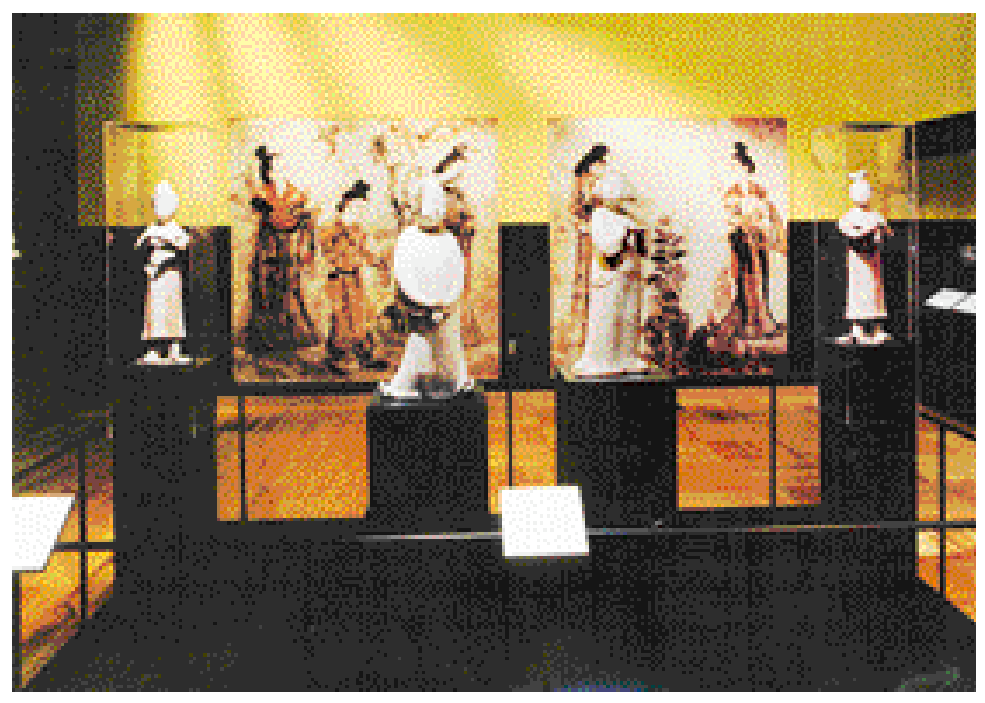

$X i$ 'an, capitale éternelle. Accompagnatrices et femmes en robe rouge, sculptures en terre cuite peinte de la dynastie des Tang. Exemple d'objets sur lesquels les visiteurs ont le plus jonglé. ○ Musée de la Civilisation, Québec/Jacques Lessard 
- la dénomination « matérielle » permet d'identifier la nature du matériau constitutif ;

- la description détaillée fouille plusieurs caractéristiques physiques de l'objet ;

- la signification apporte un éclaircissement de nature intellectuelle sur le sens de l'objet, ou de l'un de ses aspects. Il s'agit d'une explication ou d'une justification de la valeur et de la portée de l'objet, ou de l'information qui précède ;

- la destination permet de connaitre l'utilité, l'utilisation ou la fonction de l'objet avant qu'il n'entre au musée ;

- la technique donne de l'information sur le procédé, le savoir-faire avec lequel l'objet a été fabriqué ;

- le contexte « individuel » de l'objet s'attache à l'évocation d'un ou de plusieurs éléments lié(s) à l'objet exposé. L’information contenue correspond à un premier degré d'abstraction par rapport à l'objet ;

- le contexte « catégoriel» de l'objet ne développe pas de sens sur l'objet présenté, mais sur la catégorie à laquelle il appartient ; - le contexte « historique » est une donnée qui précise le temps sous forme de période ou de date ;

- le contexte " géographique » est une information sur l'espace de l'objet, sa provenance, notamment ;

- le contexte « autre » est une information qui n'est pas liée à l'objet (ni de manière précise, ni de manière générale), et qui n'est pas d'ordre historique ou géographique. Il s'agit d'un élément informatif contextuel imprécis ; l'intention recherchée est générale.

Ces douze énoncés prédicatifs peuvent être regroupés en quatre types d'informations : nominatives, descriptives, explicatives et contextuelles.

Quelles différences observe t-on entre les cartels sur lesquels on compte le plus de jonglages et ceux sur lesquels les visiteurs en totalisent le moins ?

Comme le montre le tableau ci-contre, les informations nominatives, descriptives, explicatives, et contextuelles sont semblablement représentées dans les cartels sur lesquels les jonglages sont les plus nombreux. Les pourcentages relatifs à ces informations oscillent en effet entre 23 et $29 \%$ des énoncés totaux. En revanche, les pourcentages relatifs à ces mêmes informations présentent des écarts importants dans les cartels sur lesquels on compte le moins de va-etvient objet-cartel. On passe en effet du simple au triple entre les informations descriptives (12\%) et les informations contextuelles (39\%). En d'autres termes, on compte trois fois plus d'informations contextuelles que d'informations descriptives dans les cartels du groupe B. Il se peut donc qu'une répartition équilibrée des informations participe à une meilleure appréhension de l'entité objet-cartel et facilite le vaet-vient entre les deux supports. Ensuite, les énoncés descriptifs sont près de 2 fois plus présents dans les cartels du groupe A que dans les cartels du groupe $\mathrm{B}$, alors que les informations contextuelles sont une fois et demi plus présentes dans les cartels du groupe B. Il existe donc des différences notables entre ces deux groupes de cartels.

\begin{tabular}{l|c|c} 
& $\begin{array}{c}\text { Informations présentes } \\
\text { dans les cartels } \\
\text { du groupe A }\end{array}$ & $\begin{array}{c}\text { Informations présent } \\
\text { dans les cartels } \\
\text { du groupe B }\end{array}$ \\
\hline $\begin{array}{l}\text { Informations nominatives } \\
\text { Informations descriptives }\end{array}$ & $29 \%$ & $20 \%$ \\
Informations explicatives & $23 \%$ & $12 \%$ \\
Informations contextuelles & $24 \%$ & $29 \%$ \\
\hline Total & $100 \%$ & $100 \%$ \\
\hline
\end{tabular}

\section{Répartition des informations contenues dans les cartels des groupes A et B}

Le jonglage ne serait-il pas le produit d'une cohérence entre les énoncés formant l'information ? Il se peut en effet que la place de l'information dans le texte du cartel ait une incidence sur le jonglage objet-cartel. Les textes des cartels du groupe A et du groupe $\mathrm{B}$ contiennent jusqu'à 6 énoncés, 6 unités de textes ou portions de phrases. Le graphique ci-dessous représente la structuration de l'information contenue dans les cartels sur lesquels les visiteurs jonglent le plus (groupe A).

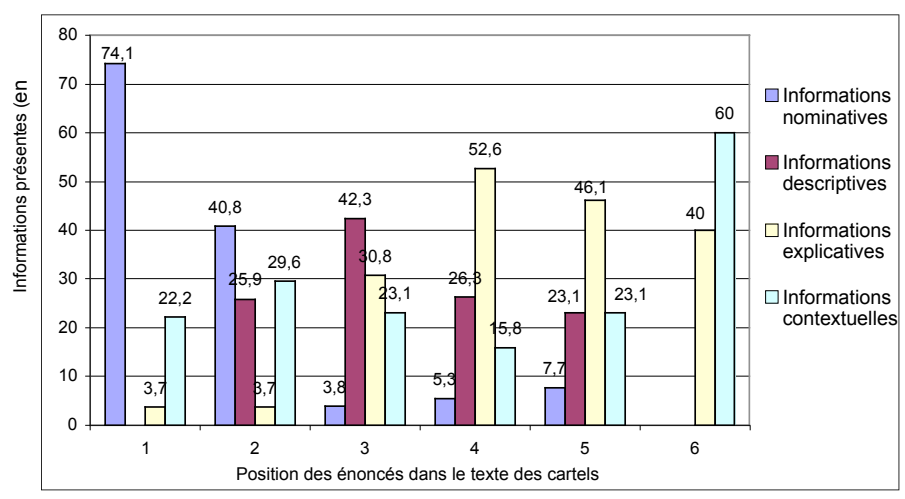

Structuration de l'information dans les cartels (cartels du groupe A) sur lesquels les visiteurs jonglent le plus (informations présentées en \%). 
Notre étude a montré que les cartels sur lesquels les visiteurs ont le moins jonglé ne possédaient pas de logique de rédaction particulière. Si toutes les informations sont potentiellement intéressantes, toutes ne sont pas en lien direct avec ce qui est montré. Afin d'illustrer nos conclusions, voici un échantillon de trois cartels prédicatifs dont la composition peut inciter au jonglage objet-cartel et conduire à une observation approfondie de l'objet. En effet, ces trois étiquettes présentent des stratégies d'écriture assez proches ; les énoncés descriptifs y ont notamment une place importante et sont susceptibles de retenir l'attention du visiteur-lecteur.

Le premier est un cartel de l'exposition Xi'an, capitale éternelle, (musée de la Civilisation, décembre 2001-septembre 2002). Il s'agit du cartel le plus utilisé par les visiteurs pour jongler avec l'objet exposé. Au-dessous du titre, des informations autonymes sont sobrement listées et précèdent un texte structuré du particulier au général, ordonnant les informations progressivement. Le cartel débute par une dénomination complète de l'objet exposé : l'objet est nommé, «vase » ; l'indication « en bronze » renseigne sur son matériau constitutif et permet de faire un renvoi aux données autonymes. Ajoutons qu'une dénomination adjectivée - telle que « cet énorme vase »- est une précision qui vient enrichir l'observation. Suit un énoncé descriptif, " à trois pieds », puis un énoncé qui informe sur la fonction de l'objet ; on mentionne en effet que le tripode servait "aux offrandes d'aliments destinées aux esprits ancestraux ». L'énoncé descriptif est ensuite repris et complété, il détaille les particularités formelles de l'objet « décoré d'un motif de crochets en 'T' entrelacés sur sa partie inférieure et d'un motif de nuages et de tonnerre sur sa partie supérieure ». En dressant un portrait précis de ce qui est donné à voir, l'information descriptive incite le visiteur à prolonger son observation, à prendre le temps de « décrypter » les caractéristiques de l'objet. Ces informations sont structurantes car elles permettent au visiteur de saisir des données plus abstraites en fin de cartel, davantage axées sur une interprétation.

\section{Tripode destiné aux offrandes d'aliments}

\section{Bronze}

\section{Zhou de l'Ouest (Xle-VIII' siècle av.J.-C.)}

Cet énorme vase en bronze à trois pieds servant aux offrandes d'aliments destinées aux esprits ancestraux est décoré d'un motif de crochets en «T» entrelacés sur sa partie inférieure et d'un motif de nuages et de tonnerre sur sa partie supérieure. Malgré ses très grosses poignées, il aurait été difficile de le soulever une fois rempli d'aliments cuits et de liquides.

Les deux cartels suivants sont extraits de l'exposition Portraits publics, portraits privés, 1770-1830, (Galeries nationales du Grand Palais, octobre 2006-janvier 2007). Tous les cartels de cette exposition présentent un texte, dont la structure est hiérarchisée et systématique. Ceux-ci commencent par une dénomination, le sujet est donc identifié. Suit un énoncé qui présente le ou la portraituré(e) et introduit le thème du tableau. Un énoncé descriptif précis d'une à deux lignes guide ensuite l'œil du regardeur, éclairant la composition des tableaux. Les énoncés descriptifs n'ont pas pour seule fonction de livrer les caractéristiques formelles du sujet peint, ils illustrent un propos scientifique plus général et toujours en lien avec le tableau exposé. Dans ce cas précis, les informations descriptives aident le visiteur à saisir les canons du portrait aristocratique anglais. Notons enfin que les cartels de cette exposition font très souvent référence à d'autres portraits exposés à proximité ; le visiteur est donc encouragé à la comparaison entre les œuvres, aux liens de sens.

\section{THOMAS GAINSBOROUGH}

\section{Sudbury, 1727 - Londres, 1788}

Giovanna Bacceli

\section{2}

Londres

Tate

Célèbre danseuse italienne, Giovanna Bacceli (vers 1757 - 1801) était alors maîtresse de Frederick Sackville, troisième duc de Dorset qui commanda ce portrait. L'artiste y interprète avec fantaisie les canons du portrait aristocratique anglais : le paysage est un décor de théâtre, le modèle est très fardé, elle exécute un mouvement de danse. Depuis Lady Alston $\left(n^{\circ} 25\right)$, la manière de l'artiste s'est faite plus libre et plus colorée.

Le troisième cartel, également extrait de Portraits publics, portraits privés, 1770-1830, est un autre exemple intéressant. Sa structure reprend les caractéristiques déjà évoquées tout en développant un élément nouveau par rapport aux deux autres : l'implication du visiteur.

En effet, en plus de justifier l'appartenance du portrait au thème de section « portrait de famille, entre sensibilité et expression d'un ordre politique ou moral », les informations descriptives de ce cartel, synthétisées et généralisées dans la dernière phrase, associent le lecteur à la démarche scientifique du concepteur. Une fois les énoncés descriptifs saisis le visiteur est en mesure de comprendre ce qui lie ce tableau à l'école de David.

\section{France, vers 1810}

Portrait d'un homme et de ses enfants

\section{Le Mans, Mussé de Tessé}

Ce portrait dont les modèles demeurent inconnus, exalte les valeurs de la bourgeoisie de la société postrévolutionnaire. Le père, magnifié comme chef de famille et exemple moral, apparaît comme le garant de l'éducation de ses enfants. Le fils ainé, élégamment mis, s'affirme par son geste protecteur, comme un digne héritier.

Le réalisme descriptif, la qualité du dessin et la rigueur de la composition situent ce tableau dans l'entourage de David.

Si l'attention accordée à la typographie, à la calligraphie, à la mise en page, au rapport entre couleur du texte et couleur du fond, à l'emplacement et l'éclairage des cartels, il semble tout aussi déterminant de veiller à la structuration des informations, dont le contenu peut faire varier le comportement du visiteur face à l'objet. 
Nous constatons tout d'abord que $74 \%$ des fragments d'énoncés qui sont en première position et $40 \%$ des énoncés placés en seconde place dans les cartels du groupe $\mathrm{A}$, sont de natures dénominatives. Les cartels sur lesquels les visiteurs jonglent le plus commencent par identifier l'objet auquel ils se rapportent. Ensuite, nous observons que les énoncés qui se positionnent en troisième place sont majoritairement de nature descriptive $(42,3 \%)$. Puis, près de la moitié des quatrième et cinquième énoncés sont explicatifs $(52,6 \%$ et $46,1 \%$ précisément). Enfin, les deux tiers des énoncés positionnés en fin de cartel permettent d'inscrire l'objet dans un cadre contextuel élargi. Les cartels qui incitent le plus au jonglage offrent donc un ensemble d'informations réparties de manière relativement homogène, constante, et hiérarchisée du particulier au général. Ils possèdent une logique rédactionnelle qui va de l'objet à son contexte.

En ce qui concerne le groupe de cartels sur lesquels les visiteurs produisent le moins de jonglages, le graphique ci-dessous, montre une toute autre structuration de l'information scientifique.

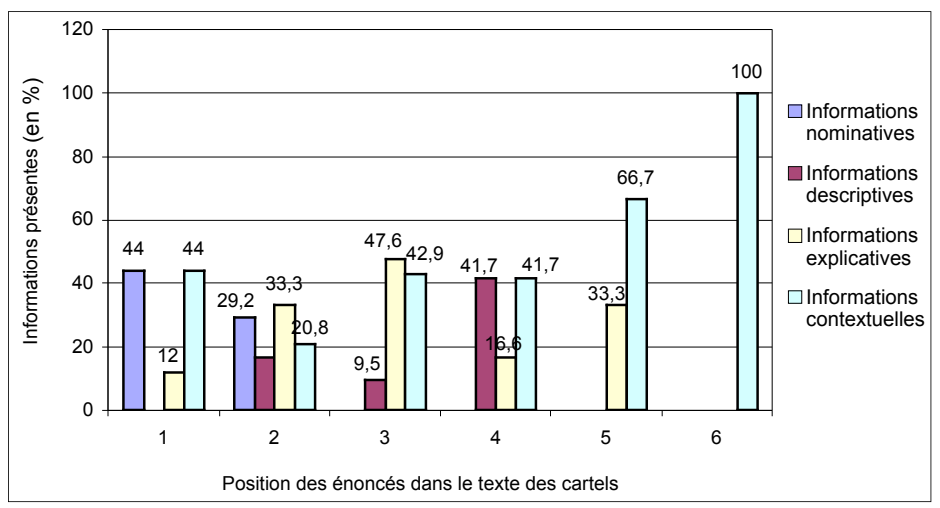

Structuration de l'information dans les cartels (cartels du groupe B) sur lesquels les visiteurs jonglent le moins.

On observe que les informations placées en $1^{\text {ere }}$ position dans le texte des cartels contiennent la même proportion d'informations dénominatives et d'informations contextuelles (44\% exactement). En d'autres termes, on trouve au même niveau, en début de cartel, des informations qui sont proches de l'objet puisqu'elles le nomment, et des données éloignées de ce qui est présenté, le propos étant également placé dans un contexte élargi. Ces deux types d'informations sont paradoxales. La rédaction des cartels sur lesquels les visiteurs jonglent le moins n'est donc pas constante. Cette interprétation corrobore les conclusions de Chandler Screven selon lequel
« les abstractions et les généralisations ne doivent pas être placées dans les titres et les paragraphes initiaux » (2), le contenu de l'étiquette devant d'abord se concentrer sur l'objet. Il se peut qu'un tel contraste de rédaction d'un cartel à l'autre, freine la compréhension de l'objet, et empêche le visiteur de cerner la logique rédactionnelle du scripteur. Cela est renforcé par le fait que l'ordre du discours ne soit jamais systématique. L'information contextuelle, par exemple, se retrouve en première, deuxième, troisième, quatrième, cinquième et sixième position. Les cartels qui suscitent le moins de jonglages offrent donc un ensemble d'informations non hiérarchisées et réparties de manière apparemment contingente et indéterminée.

Comme nous venons de le voir, le va-et-vient objetcartel est induit par la logique rédactionnelle qui régit les informations dans le texte du cartel. Souhaitant approfondir ces résultats, nous avons analysé la structure des énoncés contenus dans les deux groupes de cartels, en fonction de leur lien avec l'objet auquel ils se rapportent.

Peut-on dire qu'une étiquette qui parle de l'objet auquel elle se rapporte à plus de chance d'inciter au jonglage qu'une étiquette qui s'en éloigne ? À partir des douze types d'énoncés préalablement décrits, et contenus dans les textes des cartels, nous avons distingué les énoncés évoquant explicitement l'objet exposé, de ceux dont le sens est éloigné de ce qui est présenté. Ainsi, une hiérarchie à quatre niveaux, quatre degrés de proximité intellectuelle entre le texte des cartels et l'objet exposé auquel ils sont reliés, a été mise en place :

- le degré 1 regroupe les informations qui sont intellectuellement les plus proches de l'objet. Lobjet est nommé et décrit, il correspond aux énoncés suivants : " dénomination », « dénomination qualifiée » «dénomination matérielle », et « description ».

- le degré 2 regroupe des informations « assez proches de l'objet ». Ces informations sont un apport de sens, elles permettent de justifier et expliquer ce qui a précédemment été nommé ou décrit, il correspond aux énoncés suivants : " signification ", " technique ", « destination».

- le degré 3 regroupe des informations contextuelles « modérées »; c'est un degré d'abstraction plus important par rapport à l'objet. Il regroupe les informations contextuelles « individuelle » et " catégorielle »; - le degré 4 regroupe des informations abstraites; les énoncés qu'il rassemble sont très éloignés de l'objet exposé ; il rassemble les énoncés suivants : « contexte historique », « contexte géographique », « contexte autre ». Le scripteur évoque des éléments qui ne sont pas directement vérifiables à la vue de l'objet exposé. 
Le graphique ci-dessous permet de mieux saisir la répartition de ces degrés de proximité intellectuelle entre le contenu du cartel et l'objet exposé dans les textes des deux groupes de cartels analysés. Nous remarquons que les cartels sur lesquels les visiteurs ont le plus jonglé (cartels du groupe A) regroupent les plus forts pourcentages d'informations considérées comme étant «proches » de l'objet exposé (degrés 1 et 2). En revanche, les cartels sur lesquels les visiteurs ont le moins jonglé (cartels du groupe B) regroupent les plus forts pourcentages d'informations étant intellectuellement éloignées de l'objet auxquels ils se rapportent (degrés 3 et 4 ).

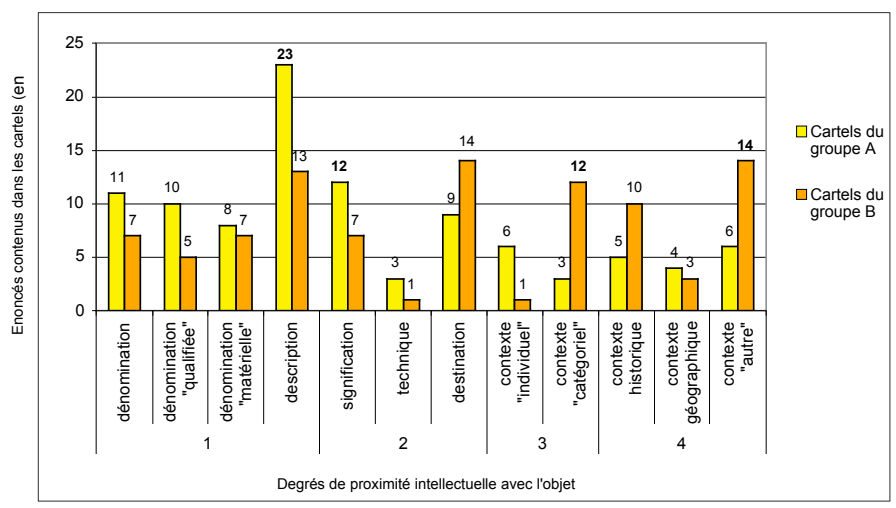

Proximité intellectuelle entre les objets et les cartels des groupes Aet B (énoncés contenus dans les cartels en \%).

Ainsi, un cartel qui parle précisément de l'objet auquel il se rapporte a plus de chance d'inciter au jonglage qu'un cartel qui s'en éloigne. En d'autres termes, un cartel présentant une logique rédactionnelle qui part de l'objet pour aller vers son contexte, et qui entretient une proximité intellectuelle avec l'objet exposé, a plus de chance d'inciter le visiteur à observer l'œuvre exposée.

En résumé, l'analyse des deux groupes de cartels a montré les points suivants :

- dans les cartels sur lesquels les visiteurs effectuent le plus de jonglages, l'objet est davantage identifié, nommé, les informations sont plus nombreuses et équitablement représentées. Celles-ci présentent des liens plus étroits avec l'objet, lequel est davantage décrit et expliqué. De plus l'objet est davantage contextualisé, et l'apport de sens y est plus important. Enfin les informations sont davantage hiérarchisées ; - dans les cartels sur lesquels les jonglages sont les plus faibles : les explications sont d'ordre plus général, le vocabulaire est moins riche (les adjectifs qualificatifs y sont moins nombreux). Enfin, les informations contextuelles sont éloignées de l'objet exposé.

\section{Structurer, hiérarchiser, systématiser}

Nous avons isolé un type d'approche de l'objet, le jonglage objet-cartel. Nous avons vu que la structuration de l'information dans le texte, et le degré de proximité intellectuelle entre ce qui est donné à voir et ce qui est donné à lire, a une forte incidence sur ce processus. Lorsqu'ils jonglent les visiteurs expriment un besoin de faire des liens entre les différents éléments de l'exposition. Cette stratégie de visite est un processus interprétatif qui peut améliorer la perception de l'objet en la rendant plus abondante, plus complexe, et en cela plus riche.

Toute approche de l'objet implique nécessairement un jonglage ; ce mouvement de va-et-vient peut prendre différentes formes. Le plus souvent, il se produit entre les référents personnels du visiteur (souvenirs, connaissances...) et l'objet exposé. Il peut également se produire entre les informations présentes dans l'exposition (dans le cartel par exemple) et l'objet exposé. Les objets muséaux sont porteurs de sens mais ces sens ne sont pas toujours «parlants » pour les visiteurs. L'exposition doit donc fournir des clés de lecture explicites et ordonnées, afin de permettre aux visiteurs qui manqueraient de référents antérieurs sur le sujet de l'exposition, de lier l'information nouvelle à ce qu'ils observent, notamment par le jonglage objet-cartel.

Pour ce faire, il semble qu'il faille accorder une attention particulière à la structuration de l'information dans les cartels en hiérarchisant les éléments informatifs du particulier au général, et en agissant de manière systématique d'un cartel à l'autre :

- structurer les informations peut faciliter le repérage des informations fondamentales,

- hiérarchiser assure un fil conducteur essentiel à l'argumentaire,

- systématiser permet au visiteur d'identifier une logique de rédaction et de suivre la progression des idées et des principes généraux de l'exposition.

Ces efforts ont toutes les chances de favoriser une approche optimale de l'objet, et par conséquent une bonne réception du média exposition.

\section{Noles}

(1) Femme 55 ans, retraitée. Extrait des entretiens réalisés après la visite de l'exposition Gratia Dei, les chemins du Moyen-Âge, (musée de la Civilisation, 2003), groupe de recherche sur les musées et l'éducation des adultes, université de Montréal.

(2) Screven, C. Comment motiver les visiteurs à la lecture des étiquettes, Publics \& Musées, ${ }^{\circ}$ 1, Paris : Presses Universitaires de Lyon, 1992, p. 42. 


\section{Bibliographie}

Bosredon, B. Les titres de tableaux : une pragmatique de l'identification. Paris : Presses Universitaires de France, 1997, 274 p.

Desjardins, J. et Jacobi, D. Les étiquettes dans les musées et les expositions scientifiques et techniques, Publics \& Musées, $n^{\circ} 1,1992$, pp. 13-31.

Dufresne-Tassé, C. et Lefebvre, A. Psychologie du visiteur de musée, contribution à l'éducation des adultes en milieu muséal. Montréal, : Hurtubise, HMH, 1995, 174 p.

Dufresne-Tassé, C., Sauvé, M., Weltz-Fairchild, A., Banna, N., Lepage, Y. et Dassa, C. Pour des expositions muséales plus éducatives, accéder à l'expérience du visiteur adulte. Développement d'une approche, Canadian Journal of Education, 23, 3, 1998a, pp. 302-316.

Dufresne-Tassé, C., Sauvé, M., Weltz-Fairchild, A., Banna, N., Lepage, Y. et Dassa, C. Pour des expositions muséales plus éducatives, accéder à l'expérience du visiteur adulte. Elaboration d'un instrument d'analyse, Canadian Journal of Education, 23, 4, 1998b, pp.421-438.

Ericcson, K.-A. et Simon, H.-A. Protocol Analysis. Cambridge : MIT Press, 1996.

Filiatrault, L. Les habiletés intellectuelles mises en œuvre au musée, in C. Dufresne-Tassé (ed.), Évaluation et éducation muséale : nouvelles tendances, Paris : Comité international de l'ICOM pour l'Éducation et l'Action culturelle, 1998, pp. 51-60.

Jacobi, D. et Poli, M.-S. Écrire/Lire les textes des étiquettes dans les musées et expositions scientifiques, in REMUS, actes du colloque sur la muséologie scientifique, Paris : Palais de la Découverte, 1991, pp. 66-75.

Kawashima, A. et Poli, M.-S. De la lecture à l'interprétation des cartels : Stratégies cognitives des visiteurs dans un musée d'art, Champs visuels, $\mathrm{n}^{\circ} 14,2000$, pp. $60-81$.

Lowe, R.-K. Améliorer l'acquisition de connaissances à partir d'objets muséaux, actes du colloque Des expositions scientifiques à l'action culturelle, des collections pour quoi faire ? Paris : Muséum national d'Histoire naturelle, 6 et 7 juillet 2000 , pp.148-161.

Meunier, A. La mise en scène des objets ethnographiques : analyse de l'influence éducative de différentes mises en exposition. Thèse de doctorat en Éducation, université d'Avignon et des Pays du Vaucluse, 2002.

Morizot, J. Interfaces : texte et image : pour prendre du recul vis-à-vis de la sémiotique. Rennes : Presses universitaires de Rennes, 2004, 130 p.
O'Neill, M.-C. Le discours d'une exposition : identification, explication, implication, in C. Dufresne-Tassé, (ed.), Évaluation et éducation muséale : nouvelles tendances, Paris : Comité international de l'ICOM pour l'Éducation et l'Action culturelle, 1998, pp. 77-87.

O’Neill, M.-C. et Dufresne-Tassé, C. Associer le visiteur de musée à la démarche scientifique comme moyen d'accroître son pouvoir d'interprétation des objets, actes du colloque Des expositions scientifiques à l'action culturelle, des collections pour quoi faire? Paris : Muséum national d'Histoire naturelle, 6 et 7 juillet 2000, pp. 236-247.

Poli, M.-S. Le parti-pris des mots dans l'étiquette : une approche linguistique, Publics \& Musées, n¹, 1992, pp. 91-103.

Poli, M.-S. Le texte au musée : une approche sémiotique. Paris : L'Harmattan, 2002, 130 p.

Serrell, B. Exhibit Labels. An interpretative approach. Walmut Creek : AltaMira Press, 1996, 260 p.

Screven, C. Comment motiver les visiteurs à la lecture des étiquettes, Publics \& Musées, $\mathrm{n}^{\circ} 1,1992$, pp. 33-53. 